\title{
Striated domains: self-organizing ordered assemblies of transmembrane $\alpha$-helical peptides and lipids in bilayers
}

\author{
Ben de Kruijff*, J. Antoinette Killian, Dragomir \\ N. Ganchev, Hilde A. Rinia a and Emma Sparr \\ Department of Biochemistry of Membranes, Institute of \\ Biomembranes, Bijvoet Center for Biomolecular \\ Research, Utrecht University, Padualaan 8, NL-3584 CH \\ Utrecht, The Netherlands \\ *Corresponding author \\ e-mail: b.dekruijff@chem.uu.nl
}

\begin{abstract}
This review summarizes the knowledge on striated domains, which are ordered assemblies of transmembrane peptides and lipids under gel-state conditions. The structure, mechanism of function and utility of this system as a model for domain formation is described, resulting in a molecular description of the domains and a discussion on the relevance of these insights for the function/formation and structure of similar domains in biological membranes.
\end{abstract}

Keywords: AFM; bacteriorhodopsin; force spectroscopy; lipid-protein interactions; phase separation; self assembly.

\section{Introduction}

The two most abundant compounds of biological membranes are lipids and proteins.

Ever since Gorter and Grendel (1925) proposed a bilayer organization for the lipids in membranes, it was realized that lipids are responsible for the most important function of membranes, to act as a barrier and to separate out from in. On the other hand, the abundance of membrane proteins is a reflection of the many other functions that membranes fulfill, such as selected transport, communication and energy conversion. What has become increasingly clear in the last decades is that the interactions between lipids and proteins are crucial for the structure, function, biogenesis and trafficking of membranes. The study of lipid-protein interactions has become a large and active field. It has recently been covered in a series of reviews (De Kruijff, 2004) and was a topic of major attention at a recent Nature Horizon sym-

\footnotetext{
Present addresses:

a Biophysics and Microscopy Group, Swammerdam Institute for Life Sciences, University of Amsterdam, Kruislaan 316, NL-1098 SM Amsterdam, The Netherlands

b Physical Chemistry 1, Center for Chemistry \& Chemical Engineering, P.O. Box 124, S-22100 Lund, Sweden
}

posium (http://www.nature.com/horizon/livingfrontier/ index.html).

One aspect of lipid-protein interactions that deserves special attention is their role in membrane domain formation. Membrane domains in which specific proteins and lipids co-segregate from the remainder of the membrane are supposed to play essential roles in processes such as signal transduction and membrane flow. A special case is represented by highly ordered membrane domains in which the components are organized in a semi-crystalline manner. The classical example is that of the purple membrane, in which bacteriorhodopsin together with specific lipids forms a highly ordered twodimensional lattice, well separated from the rest of the bacterial cytoplasmic membrane (Stoeckenius and Bogomolni, 1982). Recently it was shown that this organization plays an important role in the long-term efficiency of the photo-cycle carried out by bacteriorhodopsin (D. Oesterhelt, personal communication). Another example is the S-layer in Archaea bacteria (Grogan, 1996). The factors underlying the formation of lipid-protein domains in general and the more specialized two-dimensional lattices in particular are not well understood, but are assumed to be the result of a balance of protein-protein, proteinlipid and lipid-lipid interactions. From results of an AFM study, Mou et al. (1996) reported that the transmembrane $\alpha$-helical gramicidin A in supported gel-state bilayers caused line-type aggregates that clustered together in ordered domains. Subsequent research established that this is a more general effect, as different transmembrane $\alpha$-helical peptides also caused the formation of line-type depressions and highly ordered domains in gel-state bilayers (Rinia et al., 2000, 2002). These ordered lipidprotein complexes were called striated domains (Rinia et al., 2000). The aim of this review is to provide the current view on the molecular organization of these domains and the way in which they are formed. Furthermore, the use of these domains as models for understanding some principles of lipid-protein interactions is indicated.

\section{Visualizing striated domains}

With the exception of the bacterial, mitochondrial and possibly the chloroplast outer membrane, the integral membrane proteins in all other membranes traverse the membrane as hydrophobic $\alpha$-helices. Therefore, hydrophobic $\alpha$-helical transmembrane peptides are commonly used as models for membrane proteins. A family of such peptides, with a core of alternating leucines and alanines flanked on either side, with membrane interface-preferring residues and with blocked $\mathrm{N}$ - and $\mathrm{C}$-termini, has been studied extensively to obtain information on the 


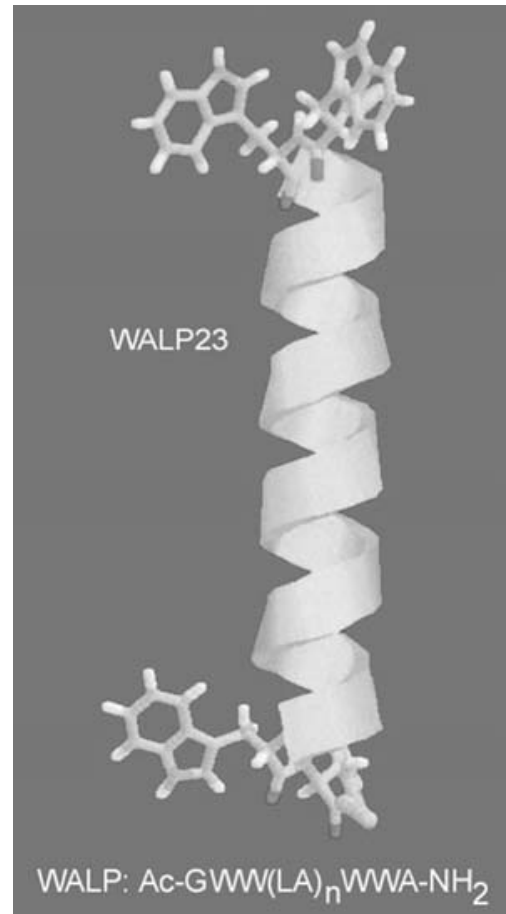

Figure 1 Schematic representation of WALP23 in $\alpha$-helical conformation and the general architecture of WALP peptides.

fundamentals of lipid-protein interactions (for a review see De Planque and Killian, 2003).

A schematic representation of WALP23, a representative of this family, is shown in Figure 1. WALP23 has a total length of 23 amino acids and a hydrophobic core flanked on both sides by two tryptophans. The presence of such peptides in supported bilayers of dipalmitoylphosphatidylcholine (DPPC) gives rise to a special surface morphology when inspected by atomic force microscopy (AFM) at room temperature (i.e., gel-state conditions) (Rinia et al., 2000).

AFM provides an image of the surface organization of bilayers when they are deposited on a solid support such as mica. Whereas a peptide-free DPPC bilayer has a flat appearance (data not shown), incorporation of 1 WALP23 per 100 DPPC molecules causes two changes in surface

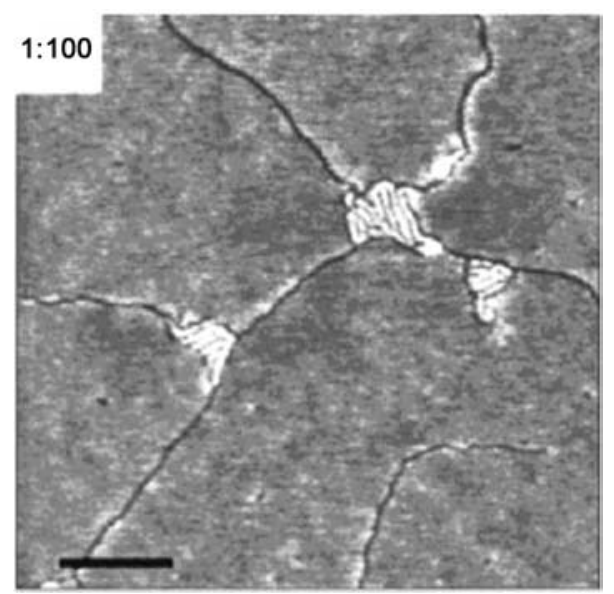

Figure 2 AFM image of $1 \mathrm{~mol} \%$ WALP23 in DPPC bilayers at room temperature.

Scale bar: $100 \mathrm{~nm}$ (for details see Rinia et al., 2000).

morphology. The presence of the peptide causes the appearance of line-type depressions, which are visible as dark (low) lines (Figure 2). These line-type depressions are bordered by slightly lighter (higher) zones. Furthermore, where three line-type depressions join, small domains are present that show up as lighter (higher) areas in which dark lines are present that are regularly spaced by $8 \mathrm{~nm}$. These are the so-called striated domains. Increasing the WALP23 concentration to $5 \mathrm{~mol} \%$ (Figure 3) shows an increase in area occupied by the striated domains, which also show a further characteristic feature, in that the dark lines often intersect at approximately $120^{\circ}$. At $10 \mathrm{~mol} \%$ the entire image is occupied by the striated domain (Figure 4). The hexagonal symmetry is evidenced by a Fourier transform of the image (insert in Figure 4).

The characteristic morphology of the striated domains is not an artifact of a supported bilayer, because similar structures were also observed with freeze-fracture electron microscopy in multi-lamellar dispersions of WALP23/ DPPC mixtures quenched from room temperature (Rinia et al., 2002). In addition, the hexagonal symmetry of the striated domains cannot be the result of the hexagonal
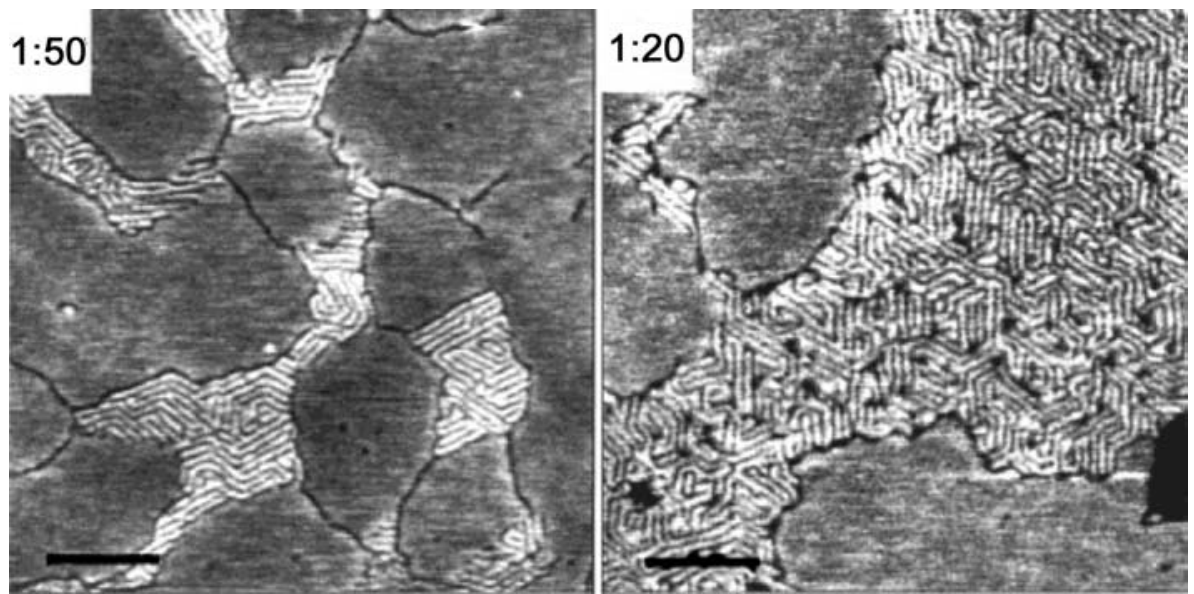

Figure 3 AFM images of 2 and 5 mol\% WALP23 in DPPC bilayers at room temperature. Scale bar: $100 \mathrm{~nm}$ (for details see Rinia et al., 2000). 


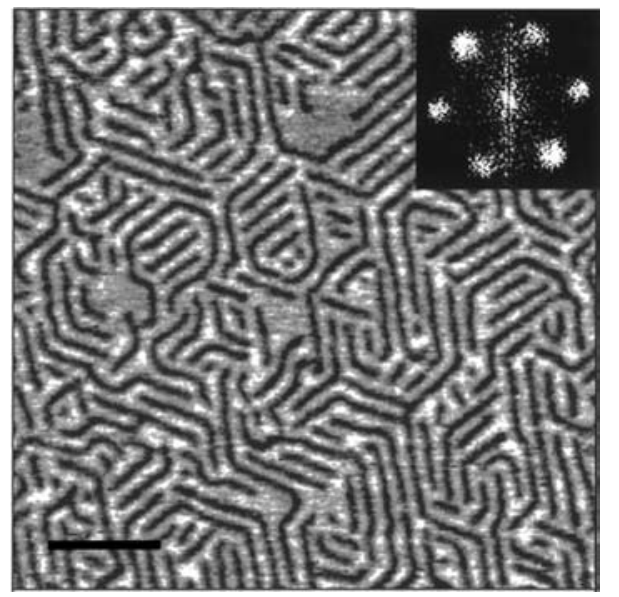

Figure 4 AFM image of $10 \mathrm{~mol} \%$ WALP23 in DPPC bilayers at room temperature.

Scale bar: $50 \mathrm{~nm}$. The inset shows a Fourier transform of the image (for details see Rinia et al., 2000).

structure of mica, because the 'repeat distance' of mica is $0.52 \mathrm{~nm}$, which is much smaller than the repeat distance of the striated domains. Striated domain formation is relatively insensitive to the length of the peptide and lipid acyl chain (Rinia et al., 2000, 2002) and is not restricted to gel-state $\mathrm{PC}$, because it also occurs for gelstate sphingomyelin (Ganchev and De Kruijff, unpublished observations). However, in the liquid ordered phase of sphingomyelin and cholesterol and in the liquid crystalline phase of PC, no domains are observed (Ganchev and De Kruijff, unpublished observations). Apparently, gel-state packing is essential for domain formation.

Domain formation is relatively insensitive to the nature of the helix flanking residues, as long as they are not positively charged. Only subtle changes in morphology are observed upon replacing the tryptophans in WALP23 for tyrosines or phenylalanines (Rinia et al., 2002). Strikingly, even the structurally completely unrelated gramicidin $A$, which spans the membrane as a dimer in a $\beta$-helical form, gives rise to morphologies that resemble the striated domains (Mou et al., 1996). However, the introduction of positively charged amino acids as flanking residues completely destroys the domains (Rinia et al., 2002), as illustrated in Figure 5 for HALP23 incorporated at $2 \mathrm{~mol} \%$ in DPPC. In HALP23 the alanine-leucine core is flanked on either side by two histidines. At $\mathrm{pH} \mathrm{9,} \mathrm{at}$ which the histidines are uncharged, typical line-type depressions and striated domains are observed in the AFM image. In contrast, at $\mathrm{pH} \mathrm{5,} \mathrm{at} \mathrm{which} \mathrm{the} \mathrm{histidines}$ carry a positive charge, the domains are completely disrupted. This suggests that electrostatic repulsions between the peptides are detrimental to domain formation, a finding that can be understood from the molecular structure of the striated domains and the way they are formed.

\section{Molecular organization of striated domains}

Based on the initial AFM detection of the peptideinduced line-type depressions and the striated domains, a molecular model was proposed for these structures (Rinia et al., 2000). The model for the line-type depressions is shown in Figure 6. To relate the model to the AFM image, a magnification of a line-type depression is shown (top left) with a height profile (top right) measured along the dark line in the AFM image. The model shows that outside the line-type depressions, a flat gel-state DPPC bilayer is present with tilted acyl chains. The height of the bilayer from the mica support is $5.8 \mathrm{~nm}$, which includes the approximately 1-nm layer of water between the support and the bilayer. The dark lines are due to linear aggregates of transmembrane peptides flanked by some disordered lipids. Between the line-type depressions and the bilayer, several DPPC molecules are still in the gel state, but have lost the tilt of their acyl chains, thereby causing an increase of approximately $0.3 \mathrm{~nm}$ in the height of the bilayer. The model for the striated domains is shown in Figure 7 with an example of an AFM image of the domain (top left) and a height profile along the dark line of the AFM image (top right). The model depicts the striated domains as a regular array of linetype depressions with a repeat distance of $8 \mathrm{~nm}$ and consisting of linear peptide aggregates flanked by some lipids that are disordered and have lost their acyl chain
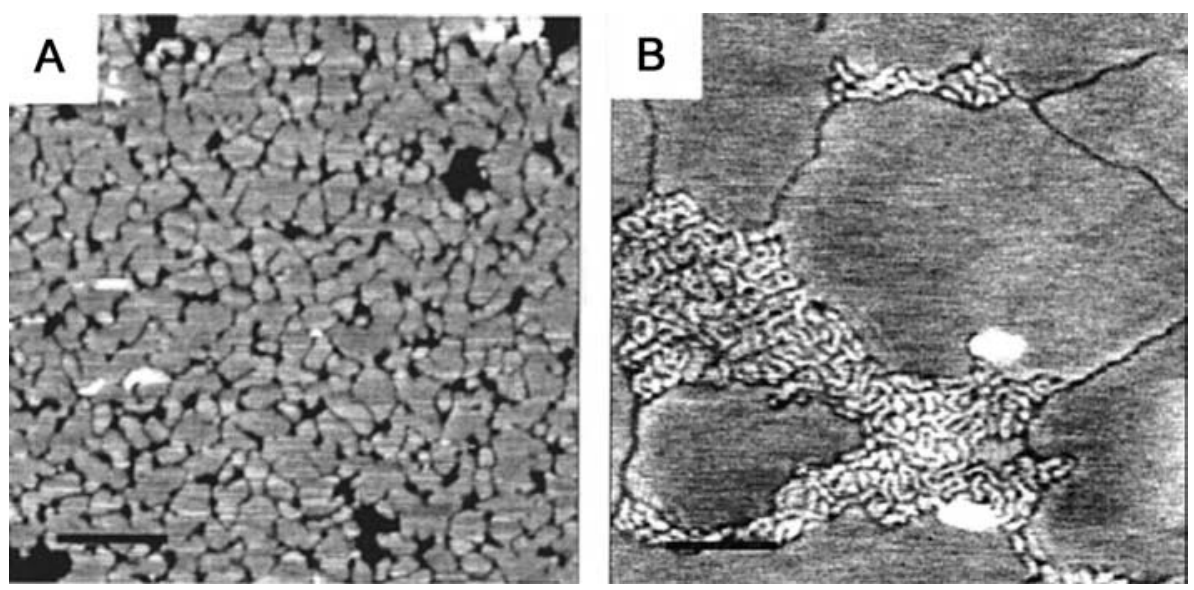

Figure 5 AFM images of 2 mol\% HALP23 in DPPC bilayers.

Conditions: (A) room temperature, $10 \mathrm{~mm}$ acetic acid, $20 \mathrm{~mm} \mathrm{NaCl}, \mathrm{pH}$ 5; (B) room temperature, $10 \mathrm{~mm}$ Tris, $20 \mathrm{~mm} \mathrm{NaCl,} \mathrm{pH} 9$. Scale bars: $200 \mathrm{~nm}(\mathrm{~A})$ and $100 \mathrm{~nm}(\mathrm{~B})$. 

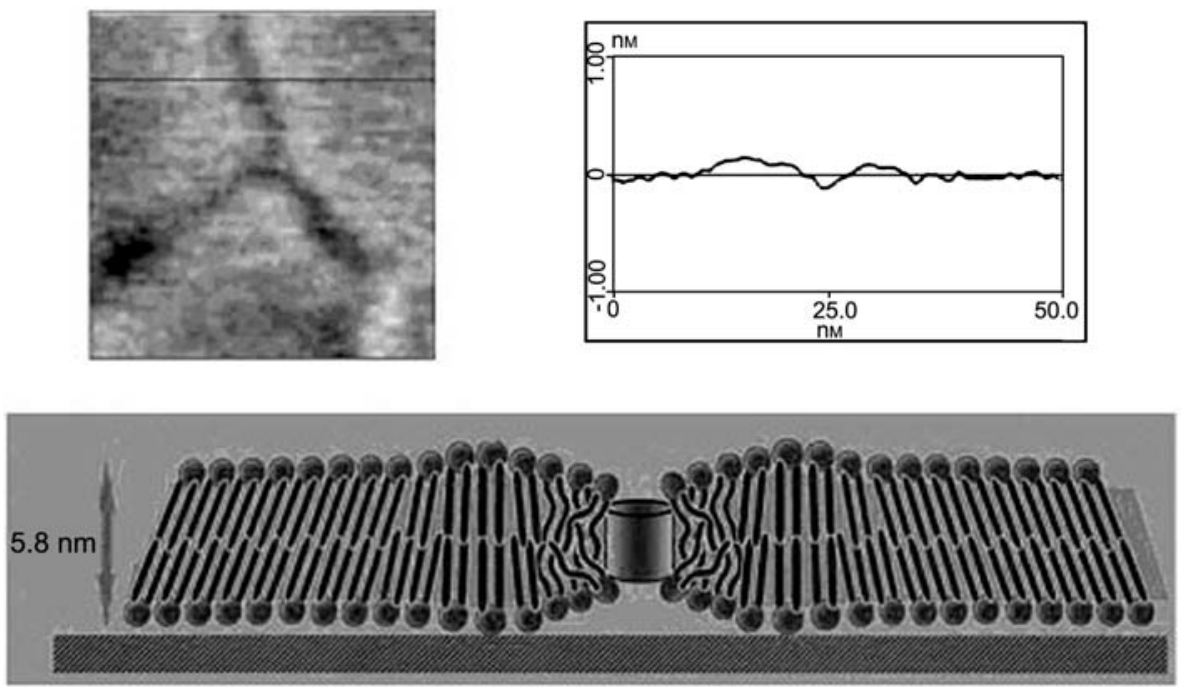

Figure 6 Molecular model of a line-type depression induced by WALP23 in supported gel-state DPPC bilayers.

The top left panel shows an enlarged image of a line-type depression as present in Figure 2. The height profile (top right) follows the black line drawn in the AFM image.

tilt. Subsequent research using multiple techniques showed that these initial models were largely correct (Sparr et al., 2005). Gold-labeling experiments showed that the peptides were localized in the line-type depressions and in the striated domains. Using photo crosslinks, fluorescence techniques and X-ray diffraction, they were shown to interact with each other (distance between the peptides $1.04 \mathrm{~nm}$ ) and with the lipid. The supposed loss of acyl chain tilt was confirmed by X-ray diffraction. Surprisingly, experiments using pyrene fluorescence-based methods with both $\mathrm{N}$ - and $\mathrm{C}$-terminal labeled peptides showed that the peptides are organized in an anti-parallel manner, as shown in a top view of the refined model of the striated domains (Figure 8). It was proposed that anti-parallel packing is favorable because it allows the helix dipoles of neighboring molecules to interact. Figure 8 shows the peptide and lipid to scale and also visualizes the hexagonal symmetry of the sys- tem. The linear aggregates of peptide make angles of $120^{\circ}$ and follow the hexagonal packing of the acyl chains of DPPC. X-Ray diffraction further showed that the hexagonal symmetry of the acyl chains is maintained in the striated domains, which suggests that it is this packing arrangement that drives the hexagonal symmetry in the striated domains. The rows of peptides are separated by approximately five rows of lipids, which suggests that the persistence length of DPPC perturbation by a row of peptides corresponds to two to three molecular layers of DPPC. The domain thus is the end result of peptide-peptide interactions in the linear peptide aggregate and lipid-peptide interactions along the peptide arrays. These insights into the organization of the line-type depressions and the striated domains, together with studies on the temperature dependence of these systems, led to a proposal of the mechanism of formation of the striated domains (Sparr et al., 2005).
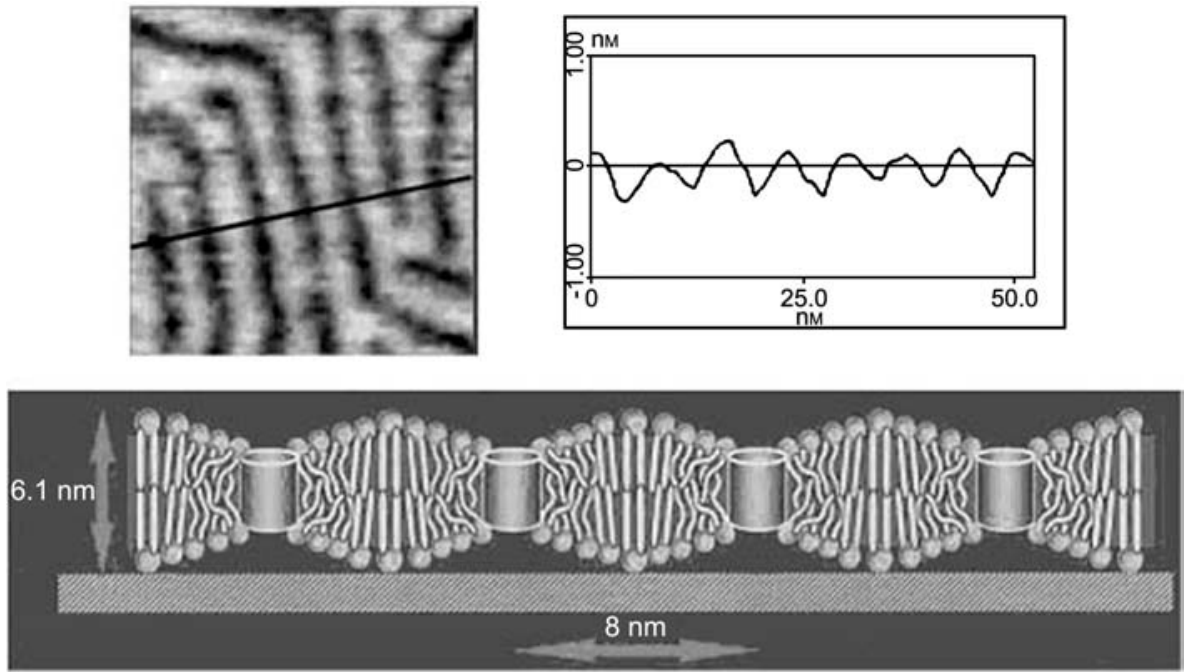

Figure 7 Molecular model of a striated domain induced by WALP23 in supported gel-state DPPC bilayers.

Top left shows an enlarged section of a striated domain as present in Figures 3 and 4 . The height profile (top right) follows the black line drawn in the AFM image. 


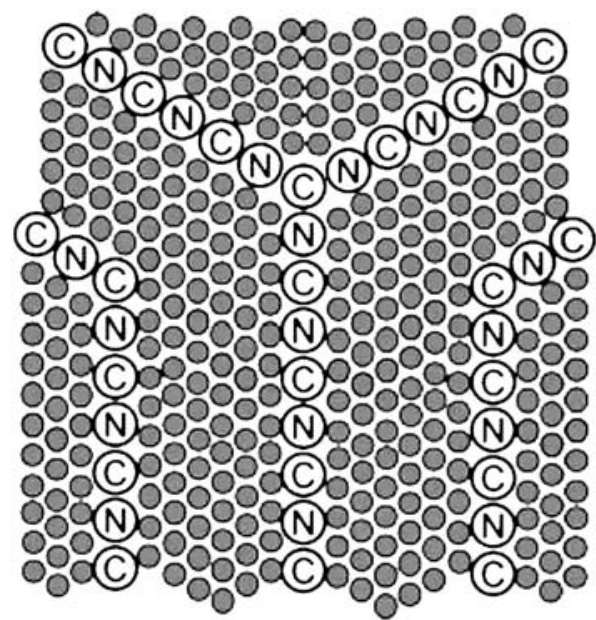

Figure 8 Refined model for the molecular organization of a striated domain.

A striated domain is shown as a top view of the bilayer, with peptides (either $\mathrm{N}$ - or $\mathrm{C}$-terminus) and lipids shown to scale.

\section{Mechanism of formation of striated domains}

When WALP23-DPPC systems containing line-type depressions and/or striated domains are heated above the chain melting temperature of the lipids (approx. $40^{\circ} \mathrm{C}$ ) all characteristic features of the striated domains are lost, but these are regained upon subsequent cooling. Figure 9 visualizes the proposed mechanism of formation of linear aggregates of WALP23 upon cooling a dilute WALP23 in DPPC system. In the melted state the transmembrane $\alpha$-helical peptides are present in a random fashion, with either the $\mathrm{N}$ - or the $\mathrm{C}$-terminus in the same bilayer water interface. The interaction between the helix dipoles is too weak to overcome the unfavorable entropy associated with the formation of antiparallel dimers or higher peptide aggregates. When the temperature is lowered to below the chain melting transition, DPPC molecules transform into the gel state with tilted chains. Freezing the acyl chains will exclude the peptides. As a result, the peptides become increasingly concentrated, thus favoring antiparallel dimer formation. The lipids interacting with the peptide are prevented from tilting and might be somewhat disordered. Gel-state formation is initiated at different sites in the bilayer, with differences in the direction of acyl chain tilt. Where such gel-state DPPC domains of different tilt directions meet, line defects are expected to occur. Such line defects in the DPPC bilayer have been experimentally observed (Grandbois et al., 1998). These line defects are the most favorable location for the WALP-DPPC (untilted) complex and at the same time they favor linear organization of the peptides, which is further stabilized by helix dipole pairing. Combining linear aggregates into striated domains is promoted because it limits unfavorable boundary effects.

The model for the line-type depressions and striated domains and the proposed mechanism for formation are consistent with many experimental observations. The relative insensitivity of the striated domains to the precise nature of the neutral helix-flanking residues and length of the peptide and acyl chains, as well as the loss of domain formation in liquid ordered or disordered systems, can be understood on the basis that the main determinant of

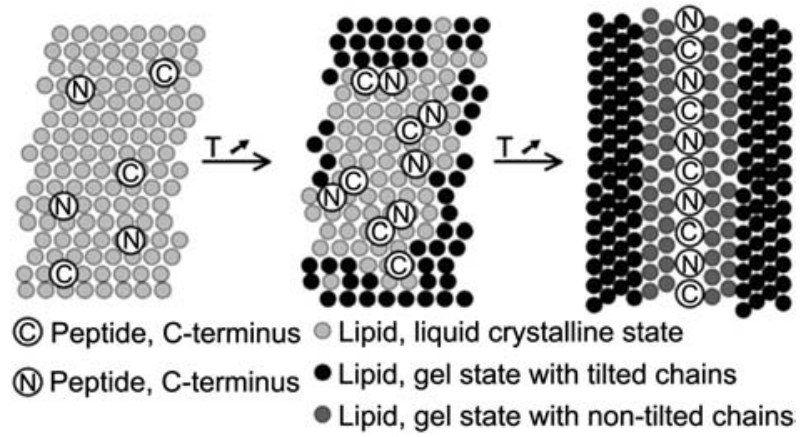

Figure 9 Proposed mechanism for the formation of onedimensional peptide arrays when a WALP23/DPPC sample is cooled to below the liquid-crystalline/gel-state transition temperature.

A top view of the bilayer is shown, starting from a liquid-crystalline state (light gray spheres) and going from an intermediate state with partly gel-state lipids with tilted chains (black spheres) to a linear array with gel-state lipids with non-tilted chains (dark gray spheres). Peptides are shown with either their $\mathrm{C}$ - or their N-terminus.

striated domain formation is gel-state formation by DPPC with hexagonal packing of acyl chains. The models also explain why transmembrane peptides with positively charged flanking residues cannot give rise to striated domain formation. This is because direct helix-helix interactions are highly unfavorable owing to electrostatic repulsion between the flanking residues of neighboring molecules. The similar repeat distance of $8 \mathrm{~nm}$ between the rows of peptides in many different striated domains can be understood as the ability of the row of transmembrane peptides to prevent the tilt of two to three molecular layers of DPPC on either site of the peptide row. This precise knowledge of the organization of striated domains is not only useful for understanding the principles of highly ordered domain formation in membranes, but also allows these domains to be used for exploring principles of lipid-protein interactions.

\section{Striated domains as systems for force microscopy}

One outstanding question in membrane research is the stability with which membrane proteins are embedded in the bilayer. A range of biophysical methods has now become available to address this question, including

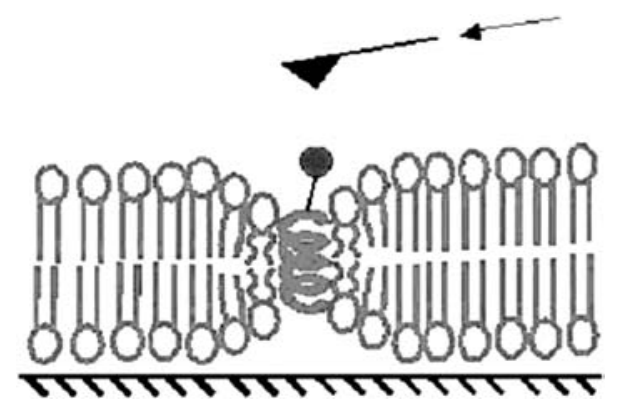

Figure 10 Schematic representation of the peptide-pulling experiment in supported gel-state DPPC bilayers, using a goldcoated AFM tip and a WALP peptide containing a thio group (dark gray sphere). For details see Ganchev et al. (2004). 


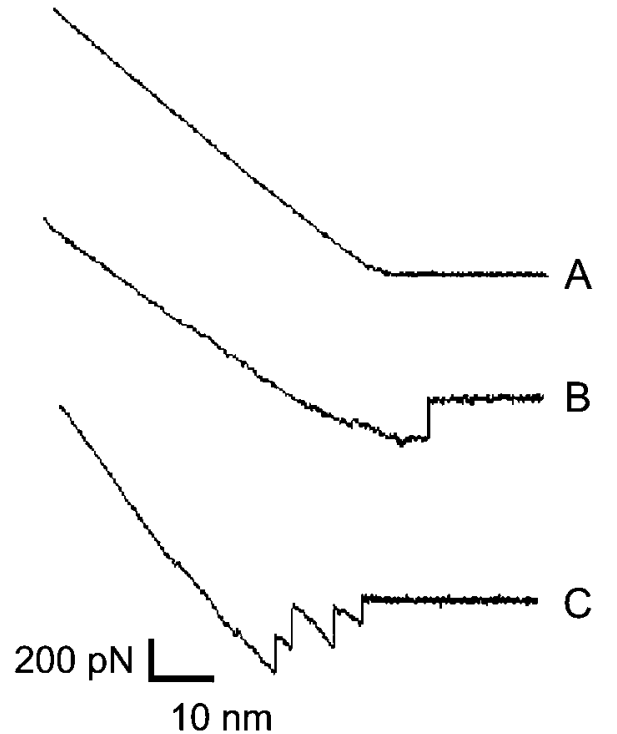

Figure 11 Representative retraction force-distance curves, recorded on different areas of a 2 mol\% SH-WALP23-containing gel-state-supported DPPC bilayer, using a gold-coated AFM tip. Curve $A$ is representative of the peptide-free domains. Curve $B$ is the typical curve for the striated domains. In rare cases, curves such as $\mathrm{C}$ are observed for the striated domains. For details see Ganchev et al. (2004).

force spectroscopy by AFM. In a pioneering study (Oesterhelt et al., 2000) gold-coated AFM tips were positioned onto the external loops of bacteriorhodopsin in its native, highly ordered, purple membrane environment. Subsequent pulling of the tip revealed the energy landscape of stepwise protein removal from the purple membrane. The same methodology has now been used on striated domains to obtain information on the removal of single $\alpha$-helices from a bilayer (Ganchev et al., 2004; Antoranz Contera et al., 2005).

The principle of the method is illustrated in Figure 10. An -SH-containing variant of WALP23 was used to create striated domains in DPPC. After visualizing the striated domain by scanning at low force, the AFM tip was positioned onto the domain, leading to the formation of a stable gold-sulfur bond. Upon subsequent pulling, removal of individual $\alpha$-helices from the striated domain could be monitored. A typical set of force curves is shown in Figure 11. Outside the domains, no interactions were measured upon retraction of the tip. However, in the majority of the cases, interactions occurred on the domains, holding the tip onto the sample. After increasing the applied pulling force, the interaction force between the peptide and the surrounding membrane was exceeded and the tip snapped back to the equilibrium position, pulling the peptide out of its hydrophobic environment. In rare cases, multiple force events were observed because of multiple attachment of peptides to the tip. It was shown that the force events measured over the striated domains correspond to the removal of individual $\alpha$-helices from the bilayer (Ganchev et al., 2004). From an analysis of the loading rate dependence of the force events, it could be concluded that the individual $\alpha$ helices are extremely strongly anchored in the bilayer. The spontaneous off-rates at room temperature were found to be $10^{-4}-10^{-5} \mathrm{~s}$, which corresponds to lifetimes of the peptide in the bilayer of hours to days. Anchoring is much more stable than for di-C16:0 lipids (Evans and Ludwig, 2000) and off-rates are only an order of magnitude higher than reported for the extremely stable biotinavidin complex (Dettmann et al., 2000). Therefore, the underlying peptide-membrane interactions represent the most stable non-covalent biological interactions reported so far and are comparable in magnitude to those reported for the removal of bacteriorhodopsin helices from the membrane (Oesterhelt et al., 2000).

Another important finding was that for peptide removal from the bilayer, a single barrier is encountered that is located $0.75 \pm 0.15 \mathrm{~nm}$ from the equilibrium state of the peptide in the striated domain. Strikingly, when pulling experiments were carried out on these SH-WALP peptides in liquid-crystalline domains of dioleoylphosphatidylcholine, very similar data were observed, which

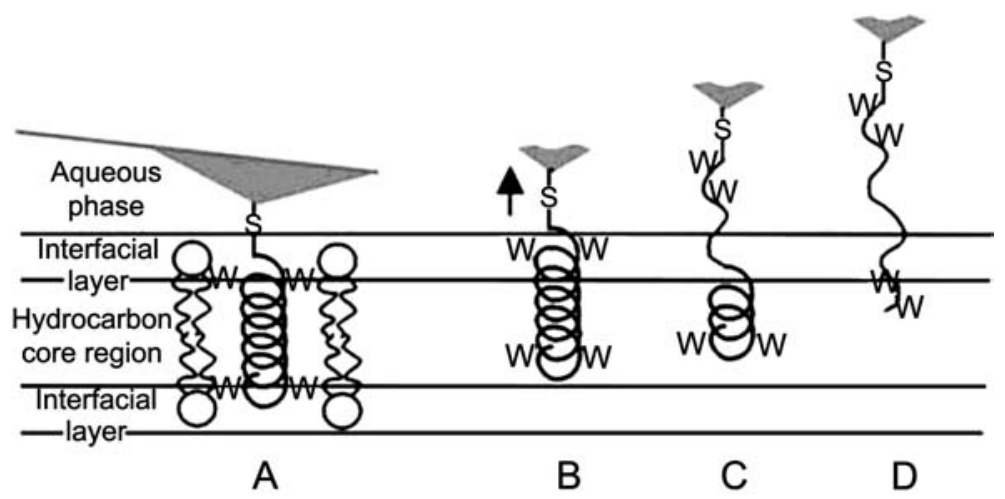

Figure 12 Schematic representation of the model describing the mechanical removal of an $\alpha$-helical transmembrane peptide (WALP23) out of the bilayer.

The domains of the peptide-lipid interface and hydrocarbon core region are approximately to scale. The gold-coated AFM tip that is chemically attached to the SH group of the peptide is not to scale. The tryptophans (W) are indicated. The peptide in the ground state is anchored in the bilayer by the tryptophans on both the cis side and the trans side and buries its hydrophobic helix into the hydrocarbon layer (A). When sufficient force is applied to the peptide such that it is displaced ca. $0.75 \mathrm{~nm}$ from its ground state, it exposes part of the hydrophobic helix to the interface (B). Because of the water present in the interface, the helix is destabilized and becomes unfolded upon pulling. At the same time, displacement of the helix from its ground state results in negative mismatch at the side facing the support, which makes incorporation of the remainder of the peptide in the bilayer less favorable (C). No further major energy barriers are expected upon further pulling, and the peptide is removed from the bilayer (D). 
demonstrates that fundamental properties of the peptide (the hydrophobic $\alpha$-helix) and the bilayer (the interfacial region) are responsible for the stability of peptide integration in the bilayer. Based on these data, a model was proposed (Ganchev et al., 2000) to describe the mechanical removal of an $\alpha$-helical peptide (WALP23) from the bilayer. The model is presented in Figure 12 and is described in its legend. The model proposes that pulling four to five residues of the hydrophobic $\alpha$-helix into the water-rich bilayer interface leads to destabilization of the helix, followed by unfolding and complete removal from the bilayer.

\section{Concluding remarks}

Striated domains formed by simple transmembrane peptides and a simple gel-state lipid, like DPPC, have proven to be interesting and valuable systems for understanding the formation of highly ordered, semi-crystalline lipid-peptide lattices. Thorough understanding of the way in which these systems are organized and formed has led to insights into the way concerted peptide-peptide, peptide-lipid and lipid-lipid interactions can lead to the formation of large and uniquely structured membrane domains. A striking parallel can be drawn between striated domains and the purple membrane in which bacteriorhodopsin is localized. The purple membrane also consists of a very regular lattice of bacteriorhodopsin and ordered lipids that are present in a system with hexagonal symmetry. The insight generated by studies of striated domains will contribute to understanding the assembly of the purple membrane and related highly ordered membrane domains. Studies on striated domains suggest that ordered lipids play an important role in determining the symmetry of the purple membrane. Striated domains are also promising model systems for analysis of the fundamental aspects of lipidprotein interactions. They have been valuable in analyzing the stability of integration of $\alpha$-helices in bilayers, provided evidence for antiparallel helix pairing, and have given insight into the persistence length of peptidelipid interaction and the stability of embedding in the membrane.

\section{Acknowledgments}

The research described in this paper was supported by the NWO Division of Chemical Sciences (CW) and The Swedish Foundation for International Cooperation in Research and Higher Education (STINT). We thank R.A. Kik, R.A. Demel, M.M.E. Snel, J.P.J.M. Van der Eerden, J-W.P. Boots, D.T.S. Rijkers, A.N.J.A. Ridder, L.M.J. Kroon-Batenburg and V. Chupin for their contributions to the work on striated domains.

\section{References}

Antoranz Contera, S., Lemaître, V., De Planque, M.R.R., Watts, A., and Ryan, J.F. (2005). Unfolding and extraction of a transmembrane $\alpha$-helical peptide: dynamic force microscopy and molecular dynamics simulations. Biophys. J. 89, 3129-3140.

De Kruijff, B. (2004). Guest editor of special issue. Biochim. Biophys. Acta 1666, 1-275.

De Planque, M.R.R. and Killian, J.A. (2003). Protein-lipid interactions studied with designed transmembrane peptides: role of hydrophobic matching and interfacial anchoring. Mol. Membr. Biol. 20, 271-284.

Dettmann, W., Grandbois, M., Andre, S., Benoit, M., Wehle, A.K., Kaltner, H., Gabius, H.J., and Gaub, H.E. (2000). Differences in zero-force and force-driven kinetics of ligand dissociation from $\beta$-galactoside-specific proteins (plant and animal lectins, immunoglobulin G) monitored by plasmon resonance and dynamic single molecule force microscopy. Arch. Biochem. Biophys. 383, 157-170.

Evans, E. and Ludwig, F. (2000). Dynamic strengths of molecular anchoring and material cohesion in fluid biomembranes. J. Phys. Condens. Matter 12, A315-A320.

Ganchev, D.N., Rijkers, D.T.S., Snel, M.M.E., Killian, J.A., and De Kruijff, B. (2004). Strength of integration of transmembrane $\alpha$-helical peptides in lipid bilayers as determined by atomic force spectroscopy. Biochemistry 43, 14987-14993.

Gorter, E. and Grendel, F. (1925). On bimolecular layers of lipoids on the chromocytes of the blood. J. Exp. Med. 41, 439-443.

Grandbois, M., Clausen-Schaumann, H., and Gaub, H. (1998). Atomic force microscope imaging of phospholipid bilayer degradation by phospholipase $A_{2}$. Biophys. J. 74, 23982404.

Grogan, D.W. (1996). Organization and interactions of cell envelope proteins of the extreme thermo-acidophile Sulfolobus acidocaldarius. Can. J. Microbiol. 42, 1163-1171.

Mou, J., Czajkowsky, D.M., and Shao, Z. (1996). Gramicidin A aggregation in supported gel state phosphatidylcholine bilayers. Biochemistry 35, 3222-3226.

Oesterhelt, F., Oesterhelt, D., Pfeiffer, M., Engel, A., Gaub, H.E., and Muller, D.J. (2000). Unfolding pathways of individual bacteriorhodopsins. Science 288, 143-146.

Rinia, H.A., Kik, R.A., Demel, R.A., Snel, M.M.E., Killian, J.A., Van der Eerden, J.P.J.M., and De Kruijff, B. (2000). Visualization of highly ordered striated domains induced by transmembrane peptides in supported phosphatidylcholine bilayers. Biochemistry 39, 5852-5858.

Rinia, H.A., Boots, J-W.P., Rijkers, D.T.S., Kik, R.A., Snel, M.M.E., Demel, R.A., Killian, J.A., Van der Eerden, J.P.J.M., and De Kruijff, B. (2002). Domain formation in phosphatidylcholine bilayers containing transmembrane peptides: specific effects of flanking residues. Biochemistry 41, 2814-2824.

Sparr, E., Ganchev, D.N., Snel, M.M.E., Ridder, A.N.J.A., KroonBatenburg, L.M.J., Chupin, V., Rijkers, D.T.N., Killian, J.A., and De Kruijff, B. (2005). Molecular organization in striated domains induced by transmembrane $\alpha$-helical peptides in dipalmitoyl phosphatidylcholine bilayers. Biochemistry 44 , 2-10.

Stoeckenius, W. and Bogomolni, R.A. (1982). Bacteriorhodopsin and related pigments of halobacteria. Annu. Rev. Biochem. $51,587-616$. 